\title{
A note on some fundamental results in complete gauge spaces and application
}

\author{
Mohamed Jleli ${ }^{1}$, Bessem Samet ${ }^{1 *}$, Calogero Vetro ${ }^{2}$ and Francesca Vetro ${ }^{3}$
}

\author{
"Correspondence: \\ bsamet@ksu.edu.sa \\ ${ }^{1}$ Department of Mathematics, King \\ Saud University, Riyadh, Saudi \\ Arabia \\ Full list of author information is \\ available at the end of the article
}

\begin{abstract}
We discuss the extension of some fundamental results in nonlinear analysis to the setting of gauge spaces. In particular, we establish Ekeland type and Caristi type results under suitable hypotheses for mappings and cyclic mappings. Our theorems generalize and complement some analogous results in the literature, also in the sense of ordered sets and oriented graphs. We apply our results to establishing the existence of solution to a second order nonlinear initial value problem.
\end{abstract}

MSC: 47H10; 54A20; 34L30

Keywords: gauge structure; fixed point; monotone operator; ordinary differential equation

\section{Introduction}

The variational principle established by Ekeland in 1972, see [1, 2], is one of the most discussed and applied result in the context of nonlinear analysis. This principle plays a crucial role in establishing many theoretical results. Here, we refer to the statement below.

Definition 1.1 Let $(X, d)$ be a metric space. A function $\varphi: X \rightarrow[0,+\infty)$ is lower semicontinuous at $x \in X$ if and only if, for every sequence $\left\{x_{n}\right\}$ in $X$ with $x_{n} \rightarrow x$ as $n \rightarrow+\infty$, $\liminf _{n \rightarrow+\infty} \varphi\left(x_{n}\right) \geq \varphi(x)$. Also, $\varphi$ is lower semicontinuous if and only if it is lower semicontinuous at every $x \in X$.

Now, $L(y):=\{x \in X: \varphi(x) \leq y\}$ is called the lower counter set defined by a point $y \in$ $[0,+\infty)$. Then the following results hold true.

Proposition 1.1 Let $(X, d)$ be a metric space. Let $\varphi: X \rightarrow[0,+\infty)$ be a function. Then $\phi$ is lower semicontinuous if and only if $L(y)$ is closed for every $y \in[0,+\infty)$.

Theorem 1.1 ([2]) Let $(X, d)$ be a complete metric space and $\varphi: X \rightarrow[0,+\infty]$ be a proper and lower semicontinuous function. Then, for all $c>0, \delta>0$, and $x_{0} \in X$ such that $\varphi\left(x_{0}\right) \leq$ $\inf _{x \in X} \varphi(x)+c \delta$, there exists $x^{*} \in X$ such that

(i) $\varphi\left(x^{*}\right) \leq \varphi\left(x_{0}\right)$;

(ii) $d\left(x_{0}, x^{*}\right) \leq \delta$;

(iii) $\varphi\left(x^{*}\right)<\varphi(x)+c d\left(x, x^{*}\right)$ for all $x \neq x^{*}$.

(c) 2015 Jleli et al.; licensee Springer. This article is distributed under the terms of the Creative Commons Attribution 4.0 International License (http://creativecommons.org/licenses/by/4.0/), which permits unrestricted use, distribution, and reproduction in any medium, provided you give appropriate credit to the original author(s) and the source, provide a link to the Creative Commons license, and indicate if changes were made. 
Of course, in the above statement we can, alternatively, consider a function $\varphi: X \rightarrow$ $(-\infty,+\infty]$, by adding the hypothesis that it is bounded from below. The other fundamental theorem that we would like to discuss is the following fixed point result established by Caristi in 1976; see [3].

Theorem 1.2 ([3]) Let $(X, d)$ be a complete metric space and $f: X \rightarrow X$ be a mapping not necessarily continuous. Assume that there exists a function $\varphi: X \rightarrow[0,+\infty)$, which is lower semicontinuous, such that

$$
d(x, f x) \leq \varphi(x)-\varphi(f x) \quad \text { for all } x \in X
$$

Then $f$ has a fixed point $z$, that is, $z=f z$.

Also, $f$ is called a Caristi mapping on $(X, d)$. The above theorems are strongly related each other: it is well known that the results of Ekeland and Caristi are equivalent.

On the other hand, we notice that most of the spaces studied in mathematical analysis, share many algebraic and topological properties as well as metric properties. Consequently, there is no line separating clearly metric theory from the other topological or set-theoretic branches. In view of this fact, many authors considered the problem of establishing theoretic results of nonlinear analysis in a metric space (see, for instance, [4-6]). On the other hand, since several notions and theorems in the literature do not require that all the properties of a metric hold true, various definitions of generalized metrics were introduced (see, for example, $[7,8]$ ). Here we are interested in the so-called gauge spaces that are characterized by the fact that the distance between two points of the space may be zero even if the two points are distinct. For instance, Frigon [9, 10], Chiş and Precup [11] gave generalizations of fixed point theorems and Ekeland's variational principle on gauge spaces (see also [12-15]). Consistent with this line of research, our aim is to further discuss the above fundamental theorems, by establishing new results under modified conditions in complete gauge spaces. In particular, we deal with ordered sets and oriented graphs. Then, to illustrate the usefulness of our theory, we apply our results to establishing the existence of a solution to a second order nonlinear initial value problem.

\section{Preliminaries}

We collect some preliminaries on gauge spaces and basic definitions.

Definition 2.1 Let $X$ be a nonempty set. A function $d: X \times X \rightarrow[0,+\infty)$ is called a pseudo-metric in $X$ whenever

(i) $d(x, x)=0$ for all $x \in X$;

(ii) $d(x, y)=d(y, x)$ for all $x, y \in X$;

(iii) $d(x, y) \leq d(x, z)+d(z, y)$ for all $x, y, z \in X$.

Definition 2.2 Let $X$ be a nonempty set endowed with a pseudo-metric $d$. The $d$-ball of radius $\varepsilon>0$ centered at $x \in X$ is the set

$$
B(x ; d, \varepsilon)=\{y \in X \mid d(x, y)<\varepsilon\} .
$$


Definition 2.3 A family $\mathcal{F}=\left\{d_{\lambda} \mid \lambda \in \mathcal{A}\right\}$ of pseudo-metrics is called separating if for each pair $(x, y)$ with $x \neq y$, there is a $d_{\lambda} \in \mathcal{F}$ such that $d_{\lambda}(x, y) \neq 0$.

Definition 2.4 Let $X$ be a nonempty set and $\mathcal{F}=\left\{d_{\lambda} \mid \lambda \in \mathcal{A}\right\}$ be a family of pseudometrics on $X$. The topology $\mathcal{T}(\mathcal{F})$ having as a sub-basis the family

$$
\mathcal{B}(\mathcal{F})=\left\{B\left(x ; d_{\lambda}, \varepsilon\right) \mid x \in X, d_{\lambda} \in \mathcal{F}, \varepsilon>0\right\}
$$

of balls is called the topology in $X$ induced by the family $\mathcal{F}$. The pair $(X, \mathcal{T}(\mathcal{F}))$ is called a gauge space. Notice that $(X, \mathcal{T}(\mathcal{F}))$ is Hausdorff if we require that $\mathcal{F}$ is separating.

Definition 2.5 Let $(X, \mathcal{T}(\mathcal{F}))$ be a gauge space with respect to the family $\mathcal{F}=\left\{d_{\lambda} \mid \lambda \in \mathcal{A}\right\}$ of pseudo-metrics on $X$. Let $\left\{x_{n}\right\}$ be a sequence in $X$ and $x \in X$. Then

(a) the sequence $\left\{x_{n}\right\}$ converges to $x$ if and only if

for all $\lambda \in \mathcal{A}$ and $\varepsilon>0$, there exists $N \in \mathbb{N}$ such that $d_{\lambda}\left(x_{n}, x\right)<\varepsilon$ for all $n \geq N$. In this case, we denote $x_{n} \stackrel{\mathcal{F}}{\rightarrow} x$.

(b) The sequence $\left\{x_{n}\right\}$ is Cauchy if and only if

for all $\lambda \in \mathcal{A}$ and $\varepsilon>0$, there exists $N \in \mathbb{N}$ such that $d_{\lambda}\left(x_{n+p}, x_{n}\right)<\varepsilon, \forall n \geq N, p \in \mathbb{N}$.

(c) $(X, \mathcal{T}(\mathcal{F}))$ is complete if and only if any Cauchy sequence in $(X, \mathcal{T}(\mathcal{F}))$ is convergent to an element of $X$.

(d) A subset of $X$ is said to be closed if it contains the limit of any convergent sequence of its elements.

For complete reading on gauge spaces we suggest [16]. Notice that every metric space is a pseudo-metric space. Also, if a pseudo-metric $d$ is not a metric, it is because there are at least two points $x \neq y$ for which $d(x, y)=0$. In most situations this does not happen, which means that metrics come up in mathematics more often than pseudo-metrics; however, pseudo-metrics arise in a natural way in functional analysis and in the theory of hyperbolic complex manifolds [17].

Frigon in 2011, see [10], proved useful generalizations of the Ekeland variational principle and Caristi's fixed point theorem in complete gauge spaces. However, in establishing her results, she does not require that the family $\mathcal{F}$ is separating, but she uses a gauge structure $\left\{d_{n} \mid n \in \mathbb{N}\right\}$ satisfying the following condition:

$$
d_{1}(x, y) \leq d_{2}(x, y) \leq \cdots \quad \text { for all } x, y \in X
$$

Theorem 2.1 ([10]) Let $X$ be endowed with a complete gauge structure $\left\{d_{n} \mid n \in \mathbb{N}\right\}$ satisfying condition (1). For every $n \in \mathbb{N}$, let $\varphi_{n}: X \rightarrow[0,+\infty]$ be a proper and lower semicontinuous function. Then, for all sequences of positive numbers $\left\{c_{n}\right\},\left\{\delta_{n}\right\}$, and $x_{0} \in X$ such that $\varphi_{n}\left(x_{0}\right) \leq \inf _{x \in X} \varphi_{n}(x)+c_{n} \delta_{n}$, there exists $x^{*} \in X$ such that

(i) $\varphi_{n}\left(x^{*}\right) \leq \varphi_{n}\left(x_{0}\right)$ for all $n \in \mathbb{N}$;

(ii) $d_{n}\left(x_{0}, x^{*}\right) \leq \delta_{n}$ for all $n \in \mathbb{N}$;

(iii) for all $x \neq x^{*}$, there exists $n \in \mathbb{N}$ such that $\varphi_{n}\left(x^{*}\right)<\varphi_{n}(x)+c_{n} d_{n}\left(x, x^{*}\right)$. 
Theorem 2.2 ([10]) Let $X$ be endowed with a complete gauge structure $\left\{d_{n} \mid n \in \mathbb{N}\right\}$ satisfying condition (1). Let $f: X \rightarrow X$ be a mapping. For every $n \in \mathbb{N}$, let $\varphi_{n}: X \rightarrow[0,+\infty)$ be a lower semicontinuous function such that

$$
d_{n}(x, f x) \leq \varphi_{n}(x)-\varphi_{n}(f x) \quad \text { for all } x \in X
$$

Then $f$ has a fixed point $z$, that is, $z=f z$.

\section{Main results}

\subsection{Some consequences of Frigon's theorems}

Inspired by the significant work of Frigon [10], we give some consequences of Theorem 2.2 .

Theorem 3.1 Let $X$ be endowed with a complete gauge structure $\left\{d_{n} \mid n \in \mathbb{N}\right\}$ satisfying condition (1). Let $T, f: X \rightarrow X$ be two mappings with $T$ continuous. For every $n \in \mathbb{N}$, let $r_{n}$ be a negative real number such that

$$
d_{n}(x, T f x) \leq d_{n}(x, T x)+r_{n} d_{n}(x, f x) \quad \text { for all } x \in X .
$$

Then $f$ has a fixed point $z$, that is, $z=f z$.

Proof The continuity of $T$ implies that the function $\varphi_{n}: X \rightarrow[0,+\infty)$ defined by

$$
\varphi_{n}(y):=-\frac{1}{r_{n}} d_{n}(y, T y) \quad \text { for all } y \in X,
$$

is lower semicontinuous. From (2), we get

$$
d_{n}(x, f x) \leq \varphi_{n}(x)-\varphi_{n}(f x) \quad \text { for all } x \in X
$$

Thus, by Theorem 2.2, $f$ has a fixed point.

Theorem 3.2 Let $X$ be endowed with a complete gauge structure $\left\{d_{n} \mid n \in \mathbb{N}\right\}$ satisfying condition (1). Let $f: X \rightarrow X$ be a mapping. For every $n \in \mathbb{N}$, let $k_{n} \in[0,1)$ be such that

$$
d_{n}\left(f x, f^{2} x\right) \leq k_{n} d_{n}(x, f x) \quad \text { for all } x \in X .
$$

If one of the following conditions holds:

(i) the function $h_{n}: X \rightarrow[0,+\infty)$ defined by $h_{n}(x):=d_{n}(x, f x)$ is lower semicontinuous;

(ii) the mapping $f$ is continuous;

then $f$ has a fixed point in $X$.

Proof Note that (ii) implies (i). In fact, let $x \in X$ and $\left\{x_{m}\right\} \subset X$ such that $x_{m} \rightarrow x$ as $m \rightarrow$ $+\infty$ and assume that $f$ is continuous. From

$$
\begin{aligned}
h_{n}(x) & =d_{n}(x, f x) \leq d_{n}\left(x, x_{m}\right)+d_{n}\left(x_{m}, f x_{m}\right)+d_{n}\left(f x_{m}, f x\right) \\
& =d_{n}\left(x, x_{m}\right)+h_{n}\left(x_{m}\right)+d_{n}\left(f x_{m}, f x\right),
\end{aligned}
$$


we get

$$
h_{n}(x) \leq \liminf _{m \rightarrow+\infty} h_{n}\left(x_{m}\right) .
$$

Now, we prove that $f$ has a fixed point in $X$ if (i) holds. By (3), we have

$$
d_{n}(x, f x)-k_{n} d_{n}(x, f x) \leq d_{n}(x, f x)-d_{n}\left(f x, f^{2} x\right) \quad \text { for all } x \in X .
$$

This implies that

$$
d_{n}(x, f x) \leq \varphi_{n}(x)-\varphi_{n}(f x) \quad \text { for all } x \in X
$$

where $\varphi_{n}: X \rightarrow[0,+\infty)$ is defined by

$$
\varphi_{n}(y):=\frac{1}{1-k_{n}} d_{n}(y, f y) \quad \text { for all } y \in X .
$$

Now, by (i), the function $\varphi_{n}$ is lower semicontinuous for all $n \in \mathbb{N}$. Thus, the existence of a fixed point follows by an application of Theorem 2.2.

As consequences of Theorem 3.2, we give the following results, without proof. For the origin of Theorem 3.4 and different contractive conditions, see [18, 19].

Theorem 3.3 Let $X$ be endowed with a complete gauge structure $\left\{d_{n} \mid n \in \mathbb{N}\right\}$ satisfying condition (1). Let $f: X \rightarrow X$ be a mapping. For every $n \in \mathbb{N}$, let $k_{n} \in[0,1)$ be such that

$$
d_{n}(f x, f y) \leq k_{n} d_{n}(x, y) \quad \text { for all } x, y \in X .
$$

Then $f$ has a fixed point in $X$.

Theorem 3.4 Let $X$ be endowed with a complete gauge structure $\left\{d_{n} \mid n \in \mathbb{N}\right\}$ satisfying condition (1). Let $f: X \rightarrow X$ be a mapping. For every $n \in \mathbb{N}$, let $k_{n} \in[0,1)$ be such that

$$
d_{n}(f x, f y) \leq k_{n} \max \left\{d_{n}(x, y), d_{n}(x, f x), d_{n}(y, f y), \frac{d_{n}(x, f y)+d_{n}(y, f x)}{2}\right\} \text { for all } x, y \in X .
$$

If one of the following conditions holds:

(i) the function $h_{n}: X \rightarrow[0,+\infty)$ defined by $h_{n}(x):=d_{n}(x, f x)$ is lower semicontinuous;

(ii) the mapping $f$ is continuous;

then $f$ has a fixed point in $X$.

Example 3.1 Let $X=\mathbb{R}$ and, for any $n \in \mathbb{N}$, define

$$
d_{n}(x, y)=\frac{n}{n+1}\left|x^{2}-y^{2}\right| \quad \text { for all } x, y \in X .
$$

Clearly, $\left\{d_{n} \mid n \in \mathbb{N}\right\}$ is a complete gauge structure satisfying condition (1). Also, define $f: X \rightarrow X$ by $f x=\frac{x}{2}$ for all $x \in X$. Now, for every $n \in \mathbb{N}$, let $k_{n} \in\left[\frac{1}{4}, 1\right)$ so that condition (4) is satisfied for all $x, y \in X$. Therefore, $f$ has a fixed point in $X$; here 0 is a unique fixed point of $f$. 


\subsection{Results for cyclic mappings}

In [20], Kirk et al. obtained extensions of well-known fixed point theorems for cyclic mappings, by considering, for instance, a cyclical contractive condition as given by the next theorem.

Definition 3.1 Let $A, B$ be two nonempty subsets of a metric space $(X, d)$. Then $f: X \rightarrow X$ is called a cyclic mapping associated to $(A, B)$ if the following conditions hold:

(i) $X=A \cup B$;

(ii) $f(A) \subseteq B$ and $f(B) \subseteq A$.

Theorem 3.5 ([20]) Let $A, B$ be two nonempty closed subsets of a metric space $(X, d)$ and $f: X \rightarrow X$ be a cyclic mapping associated to $(A, B)$. Let $k \in(0,1)$ be such that

$$
d(f x, f y) \leq k d(x, y) \quad \text { for all } x \in A \text { and } y \in B
$$

Then $f$ has a unique fixed point in $A \cap B$.

Inspired by this result, other fixed point theorems with cyclical contractive conditions were obtained (see, for instance, [21-23]). Our aim in this section is to prove some fixed point theorems for cyclic mappings in complete gauge spaces. First, we state the extension of Theorems 3.3 and 3.5 for a cyclic mapping and a complete gauge structure.

Theorem 3.6 Let $X$ be endowed with a complete gauge structure $\left\{d_{n} \mid n \in \mathbb{N}\right\}$ satisfying condition (1). Let $A, B$ be two nonempty closed subsets of $X$ and $f: A \cup B \rightarrow A \cup B$ be a cyclic mapping associated to $(A, B)$. For every $n \in \mathbb{N}$, let $k_{n} \in[0,1)$ be such that

$$
d_{n}(f x, f y) \leq k_{n} d_{n}(x, y) \quad \text { for all } x \in A \text { and } y \in B
$$

Then $f$ has a fixed point in $A \cap B$.

Now, we prove the following theorem.

Theorem 3.7 Let $X$ be endowed with a complete gauge structure $\left\{d_{n} \mid n \in \mathbb{N}\right\}$ satisfying condition (1). Let $A, B$ be two nonempty closed subsets of $X$ and $: A \cup B \rightarrow A \cup B$ be a cyclic mapping associated to $(A, B)$. For every $n \in \mathbb{N}$, let $\varphi_{n}^{1}: A \rightarrow[0,+\infty)$ and $\varphi_{n}^{2}: B \rightarrow[0,+\infty)$ be lower semicontinuous functions such that

$$
d_{n}(x, f x) \leq \varphi_{n}^{1}(x)-\varphi_{n}^{2}(f x) \quad \text { for all } x \in A
$$

and

$$
d_{n}(x, f x) \leq \varphi_{n}^{2}(x)-\varphi_{n}^{1}(f x) \quad \text { for all } x \in B
$$

Then $f$ has a fixed point in $A \cap B$.

Proof Let $x_{1} \in A$ and let $x_{m+1}=f x_{m}$ for all $m \in \mathbb{N}$. From (5) and (6) we get

$$
\varphi_{n}^{1}\left(x_{1}\right) \geq \varphi_{n}^{2}\left(x_{2}\right) \geq \cdots \geq \varphi_{n}^{1}\left(x_{2 m-1}\right) \geq \varphi_{n}^{2}\left(x_{2 m}\right) \geq \cdots .
$$


This implies that the sequences $\left\{\varphi_{n}^{1}\left(x_{2 m-1}\right)\right\}$ and $\left\{\varphi_{n}^{2}\left(x_{2 m}\right)\right\}$ are non-increasing and have the same limit, say $r \geq 0$. Let $p>m$. Then

$$
\begin{aligned}
d_{n}\left(x_{2 m-1}, x_{2 p}\right) & \leq \sum_{k=2 m}^{2 p} d_{n}\left(x_{k-1}, x_{k}\right) \\
& \leq \varphi_{n}^{1}\left(x_{2 m-1}\right)-\varphi_{n}^{2}\left(x_{2 m}\right)+\varphi_{n}^{2}\left(x_{2 m}\right)-\varphi_{n}^{1}\left(x_{2 m+1}\right)+\cdots+\varphi_{n}^{1}\left(x_{2 p-1}\right)-\varphi_{n}^{2}\left(x_{2 p}\right) \\
& =\varphi_{n}^{1}\left(x_{2 m-1}\right)-\varphi_{n}^{2}\left(x_{2 p}\right) \rightarrow 0 \quad(\text { as } m \rightarrow+\infty) .
\end{aligned}
$$

Since $d_{n}\left(x_{m}, x_{m+1}\right) \rightarrow 0$ as $m \rightarrow+\infty$, we see that $\left\{x_{m}\right\}$ is a Cauchy sequence and $A \cap B \neq \emptyset$. Now, we have the following:

$$
d_{n}(x, f x) \leq \min \left\{\varphi_{n}^{1}(x)-\varphi_{n}^{2}(f x), \varphi_{n}^{2}(x)-\varphi_{n}^{1}(f x)\right\} \quad \text { for all } x \in A \cap B .
$$

Thus,

$$
d_{n}(x, f x) \leq \varphi_{n}(x)-\varphi_{n}(f x) \text { for all } x \in A \cap B,
$$

where $\varphi_{n}: A \cap B \rightarrow[0,+\infty)$ is defined by $\varphi_{n}(x):=\frac{1}{2}\left(\varphi_{n}^{1}(x)+\varphi_{n}^{2}(x)\right)$ for all $x \in A \cap B$. Clearly, $\varphi_{n}$ is lower semicontinuous and, hence, the conclusion follows from Theorem 2.2.

Example 3.2 Let $A=B=X=[0,+\infty)$ and define

$$
d_{1}(x, y)= \begin{cases}0 & \text { if } x=y \text { or } x, y \in[0,1] \\ 1 & \text { otherwise }\end{cases}
$$

and, for any $n \in \mathbb{N} \backslash\{1\}$,

$$
d_{n}(x, y)= \begin{cases}d_{n-1}(x, y) & \text { if } x, y \in[0, n] \\ n & \text { otherwise }\end{cases}
$$

Clearly, $\left\{d_{n} \mid n \in \mathbb{N}\right\}$ is a complete gauge structure satisfying condition (1). Also, let $f: X \rightarrow$ $X$ be defined by

$$
f x= \begin{cases}0 & \text { if } x \notin[1,2], \\ 1 & \text { if } x \in[1,2] .\end{cases}
$$

It follows that

$$
d_{1}(x, f x)= \begin{cases}0 & \text { if } x \in[0,1] \\ 1 & \text { otherwise }\end{cases}
$$

and

$$
d_{n}(x, f x)= \begin{cases}d_{n-1}(x, f x) & \text { if } x \in[0, n] \\ n & \text { otherwise. }\end{cases}
$$


Notice that $\varphi: X \rightarrow X$, defined by

$$
\varphi(x)= \begin{cases}0 & \text { if } x \in[0,1] \\ x & \text { otherwise }\end{cases}
$$

is a lower semicontinuous function such that $d_{n}(x, f x) \leq \varphi(x)-\varphi(f x)$, for all $x \in X$. Thus, we can apply Theorem 3.7, with $\varphi_{n}^{1}=\varphi_{n}^{2}=\varphi$, to conclude that $f$ has a fixed point; here 0 and 1 are fixed points of $f$.

The following result uses a nice contractive condition introduced by Geraghty in 1973; see [24].

Theorem 3.8 Let $X$ be endowed with a complete gauge structure $\left\{d_{n} \mid n \in \mathbb{N}\right\}$ satisfying condition (1). Let $A, B$ be two nonempty closed subsets of $X$ and $f: A \cup B \rightarrow A \cup B$ be a cyclic mapping associated to $(A, B)$. For every $n \in \mathbb{N}$, let $\alpha_{n}:[0,+\infty) \rightarrow[0,1)$ be such that $\alpha_{n}\left(t_{m}\right) \rightarrow 1$ implies $t_{m} \rightarrow 0$. Assume that, for every $n \in \mathbb{N}$, the following condition holds:

$$
d_{n}(f x, f y) \leq \alpha_{n}\left(d_{n}(x, y)\right) d_{n}(x, y) \quad \text { for all } x \in A \text { and } y \in B
$$

Then $f$ has a fixed point in $A \cap B$.

Proof Let $x_{1} \in A$ and let $x_{m+1}=f x_{m}$ for all $m \in \mathbb{N}$. From (7), we deduce that

$$
d_{n}\left(x_{m+1}, x_{m+2}\right) \leq \alpha_{n}\left(d_{n}\left(x_{m}, x_{m+1}\right)\right) d_{n}\left(x_{m}, x_{m+1}\right) \leq d_{n}\left(x_{m}, x_{m+1}\right)
$$

and so the sequence $\left\{d_{n}\left(x_{m}, x_{m+1}\right)\right\}$ is non-increasing and bounded from below. This implies that there exists $r_{n} \geq 0$ such that $d_{n}\left(x_{m}, x_{m+1}\right) \rightarrow r_{n}$ as $m \rightarrow+\infty$. If $r_{n}>0$. Then, by (7), we obtain

$$
\frac{d_{n}\left(x_{m+1}, x_{m+2}\right)}{d_{n}\left(x_{m}, x_{m+1}\right)} \leq \alpha_{n}\left(d_{n}\left(x_{m}, x_{m+1}\right)\right) .
$$

Letting $m \rightarrow+\infty$, we deduce that $\alpha_{n}\left(d_{n}\left(x_{m}, x_{m+1}\right)\right) \rightarrow 1$ and so $d_{n}\left(x_{m}, x_{m+1}\right) \rightarrow 0$. To show that the sequence $\left\{x_{m}\right\}$ is Cauchy, we suppose the contrary. Assume that, given $k \in \mathbb{N}$, there exist $m>p>k$ such that

$$
d_{n}\left(x_{2 p-1}, x_{2 m}\right) \geq \varepsilon
$$

From

$$
d_{n}\left(x_{2 p-1}, x_{2 m}\right) \leq d_{n}\left(x_{2 p-1}, x_{2 p}\right)+d_{n}\left(x_{2 p}, x_{2 m+1}\right)+d_{n}\left(x_{2 m+1}, x_{2 m}\right),
$$

we get

$$
\begin{aligned}
{\left[1-\alpha_{n}\left(d_{n}\left(x_{2 p-1}, x_{2 m}\right)\right)\right] \varepsilon } & \leq\left[1-\alpha_{n}\left(d_{n}\left(x_{2 p-1}, x_{2 m}\right)\right)\right] d_{n}\left(x_{2 p-1}, x_{2 m}\right) \\
& \leq d_{n}\left(x_{2 p-1}, x_{2 p}\right)+d_{n}\left(x_{2 m+1}, x_{2 m}\right) .
\end{aligned}
$$


Letting $p, m \rightarrow+\infty$, we deduce that $\alpha_{n}\left(d_{n}\left(x_{2 p-1}, x_{2 m}\right)\right) \rightarrow 1$ and so $d_{n}\left(x_{2 p-1}, x_{2 m}\right) \rightarrow 0$. This implies that the sequence $\left\{x_{m}\right\}$ is Cauchy. Then $A \cap B \neq \emptyset$ and $x_{m} \rightarrow z \in A \cap B$. Since $f: A \cap B \rightarrow A \cap B$ is continuous, we get $z=f z$, that is, $z$ is a fixed point of $f$ in $A \cap B$.

\section{Ordered sets and oriented graphs}

In this section, we adapt the ideas in [25] to get further theorems in complete gauge spaces.

\subsection{Fixed points of monotone non-decreasing mappings}

Let $(X, \preceq)$ be a partially ordered set and $f: X \rightarrow X$ be a mapping. Then $f$ is said to be a monotone non-decreasing mapping if the following condition holds:

$$
x \preceq y \quad \Longleftrightarrow \quad f x \preceq f y \quad \text { for all } x, y \in X
$$

Also, two elements $x, y \in X$ such that $x \preceq y$ are said to be comparable.

Theorem 4.1 Let $(X, \preceq)$ be a partially ordered set endowed with a complete gauge structure $\left\{d_{n} \mid n \in \mathbb{N}\right\}$ satisfying condition (1). Let $f: X \rightarrow X$ be a continuous and monotone non-decreasing mapping. For every $n \in \mathbb{N}$, let $\varphi_{n}: X \rightarrow[0,+\infty)$ be a lower semicontinuous function such that

$$
d_{n}(x, f x) \leq \varphi_{n}(x)-\varphi_{n}(f x) \quad \text { for all } x \in X \text { with } f x \preceq x .
$$

Then $f$ has a fixed point if and only if there exists $x_{0} \in X$ with $f x_{0} \preceq x_{0}$.

Proof Let $x_{0} \in X$ with $f x_{0} \preceq x_{0}$ and let $x_{m}=f x_{m-1}$ for all $m \in \mathbb{N}$. Since $f$ is monotone nondecreasing, then $x_{m+1} \preceq x_{m}$ for every $m \in \mathbb{N}$. Therefore, for every $m, n \in \mathbb{N}$, we have

$$
d_{n}\left(x_{m}, x_{m+1}\right) \leq \varphi_{n}\left(x_{m}\right)-\varphi_{n}\left(x_{m+1}\right)
$$

This implies that the sequence $\left\{\varphi_{n}\left(x_{m}\right)\right\}$ is non-increasing and so there exists $r_{n} \geq 0$ such that $\varphi_{n}\left(x_{m}\right) \rightarrow r_{n}$ as $m \rightarrow+\infty$. For every $m, n, p \in \mathbb{N}$, we get

$$
\begin{aligned}
d_{n}\left(x_{m}, x_{m+p}\right) & \leq \sum_{i=0}^{p-1} d\left(x_{m+i}, x_{m+i+1}\right) \\
& \leq \varphi_{n}\left(x_{m}\right)-\varphi_{n}\left(x_{m+p}\right) .
\end{aligned}
$$

This implies that $\left\{x_{m}\right\}$ is a Cauchy sequence in $X$. Now, by completeness, there exists $z \in X$ such that $x_{m} \rightarrow z$ as $m \rightarrow+\infty$. Finally, by continuity of $f$ we conclude that $f z=z$, that is, $z$ is a fixed point of $f$.

On the other hand, if $x_{0}$ is a fixed point of $f$, then $x_{0}=f x_{0}$ and so the order relation $f x_{0} \preceq x_{0}$ is trivially satisfied. This completes the proof.

Remark 4.1 The novelty of the last theorem over the corresponding theorem without ordering is due to the fact that the contractive behavior of $f$ is restricted to the elements $x \in X$ which are comparable to $f x$. 


\subsection{Fixed points of $G$-edge preserving mappings}

Definition 4.1 A graph $G$ is an ordered pair $(V, E)$, where $V$ is a set and $E \subseteq V \times V$ is a binary relation. We say that $V$ is the vertex set and $E$ is the edge set.

We refer the reader to [26] for a more detailed background on this topic.

Definition 4.2 Let $G=(V, E)$ be a graph and $D$ be a subset of $V$. We say that $D$ is $G$ directed if for every $x, y \in D$, there exists $z \in V$ such that $(x, z),(y, z) \in E$.

Example 4.1 Let $V=F([0,1], \mathbb{R})$ be the set of functions $u:[0,1] \rightarrow \mathbb{R}$ and define $E \subseteq$ $V \times V$ by

$$
(u, v) \in E \quad \Longleftrightarrow u(t) \leq v(t) \quad \text { for all } t \in[0,1] .
$$

Then $G=(V, E)$ is a graph. Let $D=M([0,1], \mathbb{R})$ be the set of measurable functions $u$ : $[0,1] \rightarrow \mathbb{R}$. Then $D$ is $G$-directed. Indeed, for every $u, v \in D$, the function $z=\max \{u, v\}$ satisfies $(u, z),(v, z) \in E$.

Let $(V, d)$ be a metric space. We consider a family $G=\left\{G_{i}: 1 \leq i \leq q\right\}$ of $q \geq 1$ graphs such that $G_{i}=\left(V, E_{i}\right), E_{i} \subseteq V \times V, i=1,2, \ldots, q$.

Definition 4.3 Let $f: V \rightarrow V$ be a given mapping. We say that $f$ is $G$-monotone if for all $i=1,2, \ldots, q$, we see that $(x, y) \in E_{i}$ implies that $(f x, f y) \in E_{i+1}$, with $E_{q+1}=E_{1}$. Consequently, $\left(f^{k q} x, f^{k q} y\right) \in E_{i}$ for each nonnegative integer number $k$ if $f$ is $G$-monotone.

Remark 4.2 If $q=1\left(G=G_{1}\right)$, we say that $f$ is $G$-edge preserving; see [27].

Building on Theorem 4.1, we give a further generalization of Caristi type, by substituting the ordering relation with an oriented graph.

Theorem 4.2 Let $X$ be a complete gauge structure $\left\{d_{n} \mid n \in \mathbb{N}\right\}$ satisfying condition (1). Let $G$ be an oriented graph on $X$ such that $(x, x) \in E(G)$ for all $x \in X$. Let $f: X \rightarrow X$ be a continuous and G-edge preserving mapping. For every $n \in \mathbb{N}$, let $\varphi_{n}: X \rightarrow[0,+\infty)$ be a lower semicontinuous function such that

$$
d_{n}(x, f x) \leq \varphi_{n}(x)-\varphi_{n}(f x) \quad \text { for all } x \in X \text { with }(f x, x) \in E(G) .
$$

Then $f$ has a fixed point if and only if there exists $x_{0} \in X$ with $\left(f x_{0}, x_{0}\right) \in E(G)$.

Proof Let $x_{0} \in X$ such that $\left(f x_{0}, x_{0}\right) \in E(G)$ and let $x_{m}=f x_{m-1}$ for all $m \in \mathbb{N}$. Since $f$ is $G$ edge preserving, by Definition 4.3 and in view of Remark 4.2, we deduce that $\left(x_{m+1}, x_{m}\right) \in$ $E(G)$ for every $m \in \mathbb{N}$. Then, for every $m, n \in \mathbb{N}$, we get

$$
d_{n}\left(x_{m}, x_{m+1}\right) \leq \varphi_{n}\left(x_{m}\right)-\varphi_{n}\left(x_{m+1}\right) .
$$


This implies that the sequence $\left\{\varphi_{n}\left(x_{m}\right)\right\}$ is non-increasing and so there exists $r_{n} \geq 0$ such that $\phi_{n}\left(x_{m}\right) \rightarrow r_{n}$ as $m \rightarrow+\infty$. For every $m, n, p \in \mathbb{N}$, we have

$$
\begin{aligned}
d_{n}\left(x_{m}, x_{m+p}\right) & \leq \sum_{i=0}^{p-1} d\left(x_{m+i}, x_{m+i+1}\right) \\
& \leq \varphi_{n}\left(x_{m}\right)-\varphi_{n}\left(x_{m+p}\right) .
\end{aligned}
$$

This implies that $\left\{x_{m}\right\}$ is a Cauchy sequence in $X$. Now by completeness, there exists $z \in X$ such that $x_{m} \rightarrow z$ as $m \rightarrow+\infty$. Finally, by continuity of $f$ we conclude that $f z=z$, that is, $z$ is a fixed point of $f$.

On the other hand, if $x_{0}$ is a fixed point of $f$, then $\left(f x_{0}, x_{0}\right) \in E(G)$, since by hypothesis $(x, x) \in E(G)$ for all $x \in X$. This completes the proof.

\section{Application to ordinary differential equation}

A typical application of fixed point methods is in establishing sufficient conditions for the existence of solution of integro-differential problems. Referring to [14], we consider the following second order nonlinear initial value problem:

$$
\left\{\begin{array}{l}
x^{\prime \prime}(t)=k(t, x(t)), \quad t>0, \\
x(0)=\alpha \\
x^{\prime}(0)=\beta
\end{array}\right.
$$

where $k:[0,+\infty) \times \mathbb{R}^{n} \rightarrow \mathbb{R}^{n}$ is a continuous function. It is well known that the above problem is equivalent to the following integral equation:

$$
x(t)=\int_{0}^{t}(t-s) k(s, x(s)) d s+\beta t+\alpha, \quad t \geq 0
$$

Let $X=C\left([0,+\infty), \mathbb{R}^{n}\right)$ be the set of continuous functions defined on $[0,+\infty)$. Then, for every $n \in \mathbb{N}$, we consider the semi-norm $\|\cdot\|_{n}: X \rightarrow[0,+\infty)$ given by

$$
\|x\|_{n}=\max _{t \in[0, n]}|x(t)| \quad \text { for all } x \in X,
$$

where $|\cdot|$ denote the norm in $\mathbb{R}^{n}$. Also, for every $n \in \mathbb{N}$, let

$$
d_{n}(x, y)=\|x-y\|_{n} \quad \text { for all } x, y \in X .
$$

Clearly, $\mathcal{F}=\left\{d_{n} \mid n \in \mathbb{N}\right\}$ is a family of pseudo-metrics on $X$ satisfying condition (1). Also, $(X, \mathcal{T}(\mathcal{F}))$ is a complete gauge space.

We shall prove the following theorem.

Theorem 5.1 For every $n \in \mathbb{N}$, assume that the following condition holds:

$$
|k(s, x(s))-k(s, y(s))| \leq \gamma(s)\left[1-e^{-\min \left\{d_{n}(x, y), 1\right\}}\right] \quad \text { for each } s \in[0, n] \text { and for all } x, y \in X \text {, }
$$


and $\gamma:[0,+\infty) \rightarrow[0,+\infty)$ is such that the function $t \mapsto \int_{0}^{t}(t-s) \gamma(s) d s$ is bounded on $[0,+\infty)$ and

$$
\sup _{t \geq 0} \int_{0}^{t}(t-s) \gamma(s) d s \leq 1 .
$$

Then the second order nonlinear initial value problem (8) has a solution $x^{*} \in C([0,+\infty)$, $\left.\mathbb{R}^{n}\right)$.

Proof Consider the operator $f: X \rightarrow X$ defined by

$$
f_{x}(t)=\int_{0}^{t}(t-s) k(s, x(s)) d s+\beta t+\alpha, \quad t \geq 0, x \in X,
$$

which is well defined, since $k$ is a continuous function.

It is immediate that $x^{*}$ is a solution of (8) if and only if $x^{*}$ is a fixed point of $f$. Then we need to show that Theorem 3.8 is applicable to the operator $f$ to conclude the proof of Theorem 5.1.

Let $n \in \mathbb{N}$ and let $x, y \in X$, then for all $t \in[0, n]$ we write

$$
\begin{aligned}
|f x(t)-f y(t)| & \leq \int_{0}^{t}(t-s)|k(s, y(s))-k(s, x(s))| d s \\
& \leq \int_{0}^{t}(t-s) \gamma(s)\left[1-e^{-\min \left\{d_{n}(x, y), 1\right\}}\right] d s \\
& \leq\left[1-e^{-\min \left\{d_{n}(x, y), 1\right\}}\right] \int_{0}^{t}(t-s) \gamma(s) d s \\
& \leq 1-e^{-\min \left\{d_{n}(x, y), 1\right\}} .
\end{aligned}
$$

Then, for all $n \in \mathbb{N}$, we get

$$
d_{n}(f x, f y) \leq 1-e^{-\min \left\{d_{n}(x, y), 1\right\}} \quad \text { for all } x, y \in X,
$$

which further gives us

$$
d_{n}(f x, f y) \leq \frac{1-e^{-\min \left\{d_{n}(x, y), 1\right\}}}{\min \left\{d_{n}(x, y), 1\right\}} d_{n}(x, y) .
$$

Notice that, for every $n \in \mathbb{N}$, the function $\alpha_{n}:[0,+\infty) \rightarrow[0,1)$ given by

$$
\alpha_{n}(\tau)= \begin{cases}\frac{e^{-\min \{\tau, 1\}}-1}{-\min \{\tau, 1\}} & \text { if } \tau>0, \\ \frac{1}{2} & \text { if } \tau=0,\end{cases}
$$

is such that $\alpha_{n}\left(t_{m}\right) \rightarrow 1$ implies $t_{m} \rightarrow 0$, as $m \rightarrow+\infty$. Thus, by an application of Theorem 3.8 with $A=B=X$, we see that $f$ has a fixed point $x^{*} \in X$, that is, $x^{*} \in C\left([0,+\infty), \mathbb{R}^{n}\right)$ is a solution of (8). 
Authors' contributions

All authors contributed equally and significantly in writing this article. All authors read and approved the final manuscript.

\section{Author details}

'Department of Mathematics, King Saud University, Riyadh, Saudi Arabia. ²Dipartimento di Matematica e Informatica, Università degli Studi di Palermo, via Archirafi 34, Palermo, 90123, Italy. ${ }^{3}$ Dipartimento Energia, Ingegneria dell'Informazione e Modelli Matematici (DEIM), Università degli Studi di Palermo, viale delle Scienze, Palermo, 90128, Italy.

\section{Authors' information}

C Vetro is a member of the Gruppo Nazionale per l'Analisi Matematica, la Probabilità e le loro Applicazioni (GNAMPA) of the Istituto Nazionale di Alta Matematica (INdAM). F Vetro is a member of the Gruppo Nazionale per le Strutture Algebriche, Geometriche e le loro Applicazioni (GNSAGA) of the Istituto Nazionale di Alta Matematica (INdAM).

\section{Acknowledgements}

The authors would like to extend their sincere appreciation to the Deanship of Scientific Research at King Saud University for its funding of this research through the International Research Group Project no. IRG14-04.

\section{Received: 3 January 2015 Accepted: 17 April 2015 Published online: 29 April 2015}

\section{References}

1. Ekeland, I: On the variational principle. J. Math. Anal. Appl. 47, 324-353 (1974)

2. Ekeland, I: Sur les problèmes variationnels. C. R. Acad. Sci. Paris Sér. A-B 275, 1057-1059 (1972)

3. Caristi, J: Fixed point theorems for mappings satisfying inwardness conditions. Trans. Am. Math. Soc. 215, $241-251$ (1976)

4. Ćirić, L: A generalization of Banach principle. Proc. Am. Math. Soc. 45, 727-730 (1974)

5. Ćirić, L: Non-self mappings satisfying nonlinear contractive condition with applications. Nonlinear Anal. 71, 2927-2935 (2009)

6. Zeidler, E: Nonlinear Functional Analysis and Its Applications I: Fixed Point Theorems. Springer, Berlin (1986)

7. Freiwald, RC: Introduction to Set Theory and Topology. Lecture Notes. Washington University in St. Louis (2010)

8. Smyth, MB: Topology and Category Theory in Computer Science, pp. 207-229. Oxford University Press, New York (1991)

9. Frigon, M: Fixed point results for generalized contractions in gauge spaces and applications. Proc. Am. Math. Soc. $128,2957-2965(2000)$

10. Frigon, M: On some generalizations of Ekeland's principle and inward contractions in gauge spaces. J. Fixed Point Theory Appl. 10, 279-298 (2011)

11. Chiş, A, Precup, R: Continuation theory for general contractions in gauge spaces. Fixed Point Theory Appl. 2004(3), 173-185 (2004)

12. Agarwal, RP, O'Regan, D: Fixed-point theorems for multivalued maps with closed values on complete gauge spaces. Appl. Math. Lett. 14, 831-836 (2001)

13. Cherichi, M, Samet, B: Fixed point theorems on ordered gauge spaces with applications to nonlinear integral equations. Fixed Point Theory Appl. 2012, Article ID 13 (2012)

14. Cherichi, M, Samet, B, Vetro, C: Fixed point theorems in complete gauge spaces and applications to second order nonlinear initial value problems. J. Funct. Spaces Appl. 2013, Article ID 293101 (2013)

15. Chifu, C, Petruşel, G: Fixed-point results for generalized contractions on ordered gauge spaces with applications. Fixed Point Theory Appl. 2011, Article ID 979586 (2011)

16. Dugundji, J: Topology. Allyn \& Bacon, Boston (1966)

17. Kobayashi, S: Intrinsic metrics on complex manifolds. Bull. Am. Math. Soc. 73, 347-349 (1967)

18. Hardy, GE, Rogers, TD: A generalization of a fixed point theorem of Reich. Can. Math. Bull. 16, 201-206 (1973)

19. Reich, S: Kannan's fixed point theorem. Boll. Unione Mat. Ital. 4, 1-11 (1971)

20. Kirk, WA, Srinivasan, PS, Veeramani, P: Fixed points for mappings satisfying cyclical contractive conditions. Fixed Point Theory 4, 79-89 (2003)

21. Agarwal, RP, Alghamdi, M, Shahzad, N: Fixed point theory for cyclic generalized contractions in partial metric spaces. Fixed Point Theory Appl. 2012, Article ID 40 (2012)

22. Pacurar, M, Rus, IA: Fixed point theory for cyclic $\psi$-contractions. Nonlinear Anal. 72, 1181-1187 (2010)

23. Rus, IA: Cyclic representations and fixed points. Ann. 'Tiberiu Popoviciu' Sem. Funct. Equ. Approx. Convexity 3, 171-178 (2005)

24. Geraghty, MA: On contractive mappings. Proc. Am. Math. Soc. 40, 604-608 (1973)

25. Alfuraidan, M, Khamsi, MA: Caristi fixed point theorem in metric spaces with a graph. Abstr. Appl. Anal. 2014, Article ID 303484 (2014)

26. West, DB: Introduction to Graph Theory, 2nd edn. Prentice Hall, New York (1996)

27. Jachymski, J: The contraction principle for mappings on a metric space with a graph. Proc. Am. Math. Soc. 136, 1359-1373 (2008) 